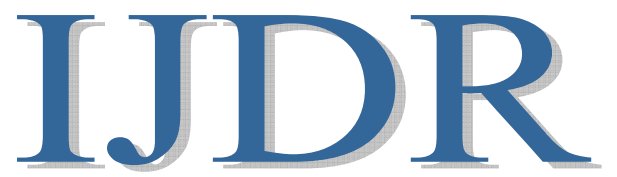

International Journal of Development Research

Vol. 10, Issue, 07, pp. 37459-37463, July, 2020

https://doi.org/10.37118/ijdr.19192.07.2020

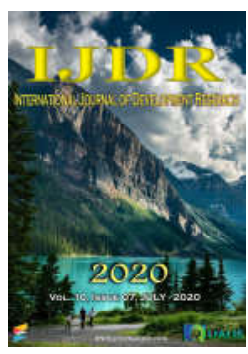

\title{
SATISFAÇÃO E ESTILO DE VIDA DE IDOSOS CORRESIDENTES
}

\section{Adrielle Santos Souza', Alba Benemérita Alves Vilela ${ }^{2}$, Lucas Silveira Sampaio ${ }^{3}$, Grazielle Marques Soares $^{4}$, Jainne Oliveira Leão Silva ${ }^{5}$, Sandiele Patez Rocha ${ }^{6}$ and Talita Santos Oliveira Sampaio ${ }^{7}$}

\author{
${ }^{1,4,5,6}$ Graduanda em Fisioterapia pela Faculdade Independente do Nordeste - FAINOR \\ ${ }^{2}$ Doutora em Enfermagem pela Universidade Federal do Ceará \\ ${ }^{3}$ Mestre em Ciências da Saúde pela Universidade Estadual do Sudoeste da Bahia \\ ${ }^{7}$ Mestre em Ciências da Saúde pela Universidade Estadual do Sudoeste da Bahia
}

\section{ARTICLE INFO}

\section{Article History:}

Received $28^{\text {th }}$ April, 2020

Received in revised form

$07^{\text {th }}$ May, 2020

Accepted $21^{\text {st }}$ June, 2020

Published online $24^{\text {th }}$ July, 2020

\section{Key words:}

Idoso. Relações Familiares. Satisfação Pessoal. Estilo de Vida.

${ }^{*}$ Corresponding author: Adrielle Santos Souza

\begin{abstract}
Objetivo: Este estudo procurou avaliar a satisfação e o estilo de vida de idosos corresidentes. Métodos: O delineamento do estudo é do tipo transversal descritivo, de base populacional e com abordagem quantitativa. Foram incluídos no estudo 239 idosos em corresidência residentes na área de abrangência das Unidades de Saúde da Família localizadas na zona urbana do município de Vitória da Conquista-Bahia. Para a coleta de dados foram utilizados a entrevista semiestruturada, o questionário Brazil old age Schedule adaptado e o Questionário sobre Estado de Saúde. A tabulação foi realizada através do software Excel 2015 e os dados foram analisados no The Statistica 1Package for Social Sciences para Windows. Resultados: Através dos dados obtidos neste estudo observou uma prevalência do sexo feminino (77\%). Os idosos corresidentes vivem em sua maioria com netos $(57,3 \%)$, seguido dos filhos $(55,2 \%)$ e filhas $(54 \%)$. No que diz respeito ao estilo de vida cerca de $(91,3 \%)$ dos entrevistados não consomem bebida alcoólica e $(52,8 \%)$ nunca fumaram. Destaca-se ainda que $(95,0 \%)$ se dizem satisfeitos com a vida. Conclusão: Através desse estudo, conclui-se que os idosos apresentam um estilo de vida favorável e boa satisfação com a vida.
\end{abstract}

Copyright (C) 2020, Adrielle Santos Souza et al. This is an open access article distributed under the Creative Commons Attribution License, which permits unrestricted use, distribution, and reproduction in any medium, provided the original work is properly cited.

Citation: Adrielle Santos Souza, Alba Benemérita Alves Vilela, Lucas Silveira Sampaio, Grazielle Marques Soares, Jainne Oliveira Leão Silva, Sandiele Patez Rocha and Talita Santos Oliveira Sampaio. "Satisfação e estilo de vida de idosos corresidentes”, International Journal of Development Research, 10, 07, $37459-37463$.

\section{INTRODUCTION}

A transição demográfica atual caracterizada pelo aumento do envelhecimento populacional provoca uma série de mudanças econômicas e sociais com repercussões em vários setores da sociedade, como por exemplo, na composição familiar. Segundo o estudo realizado pela Organização das Nações Unidas (ONU), países em desenvolvimento, como o Brasil, apresenta um grande percentual de idosos que vivem em corresidência. Neste contexto, a corresidência surge como uma das características do envelhecimento não só individual, mas também familiar, dado que, cada vez mais se identifica no seio das famílias brasileiras, a presença de pelo menos um idoso convivendo com filhos e/ou netos (CAMARANO, 2010; BARBOSA et al., 2018). Segundo afirma Torres et al. (2018), o conceito de corresidência pode ser definido como a troca de favores entre as gerações.
Esse tipo de arranjo pode ocorrer pertinente a necessidade que o idoso possa apresentar devido condição de fragilidade, que muitas vezes está associada a uma doença crônicodegenerativa ou a um quadro de comorbidade. Além disso, pode estar associada a questões financeiras, uma vez que essa relação intergeracional proporciona aos seus integrantes restrição de despesas. Esse convívio familiar estar acompanhado de benefícios e algumas dificuldades de adaptação, não só por parte do idoso, mas também pelos demais membros familiares podendo refletir na satisfação de vida dos idosos (Horta; Ferreira; Zhao, 2010). Os benefícios da corresidência estão relacionados a companhia e a segurança, a satisfação das necessidades financeiras que se apresentam como estratégia utilizada pela família, para garantir a redução dos gastos. Neste sentido, as economias geradas podem servir como um incentivo a mais para o estabelecimento deste tipo de arranjo familiar (Santos et al., 2019). 
Entretanto, esse convívio pode apresentar dificuldades, uma vez que as diferenças de ideias entre gerações podem fazer surgir conflitos, podendo inclusive gerar estresse emocional e maus tratos aos mais velhos e com isso não garantir uma satisfação e estilo de vida favorável ao idoso corresidente (Sampaio et al., 2019). Diante desse cenário torna-se importante a realização deste estudo tendo como foco a corresidência, uma vez que existe o pensamento na sociedade de que viver em família é sempre benéfico para os idosos, porém alguns estudos também analisam que viver em família pode afetar a saúde e a vida desses idosos. Observa-se também que existem poucos estudos relacionados a essa temática. Sendo assim, o estudo terá alta relevância para aprofundamento de conhecimentos sobre a satisfação e o estilo de vida dos idosos no cenário atual, contribuindo, assim, para elaboração de ações de promoção e prevenção a saúde e intervenções por meio de profissionais dessa área, atendendo as necessidades e contribuindo para melhoria do estilo de vida desses idosos. Deste modo, a pesquisa tem por objetivo avaliar a satisfação e o estilo de vida de idosos corresidentes.

\section{MATERIAIS E MÉTODOS}

Trata-se de um estudo descritivo e exploratório com delineamento transversal e abordagem quantitativa. Esta pesquisa faz parte do projeto intitulado "Arranjo Familiar De Idosos Residentes Em Municípios Do Nordeste E Sudeste Do Brasil" o qual foi realizado por meio de uma proposta de pesquisa no município de Salto da Divisa-MG, Jequié-BA e Vitória da Conquista-Ba. Entretanto, destaca-se, que para o presente estudo utilizou-se dados apenas do município de Vitória da Conquista-BA. Estando vinculado ao Núcleo de Pesquisa e Estudos em Saúde do Idoso - NUPESI, da Faculdade Independente do Nordeste - FAINOR. Foi desenvolvido em duas Unidades de Saúde da Família (USF) do município de Vitória da Conquista - BA, que foram selecionadas através de um sorteio simples. Foram incluídos na população do estudo todos os idosos em situação de corresidência cadastrados nas Unidades de Saúde da Família, totalizando 239 idosos. Considera-se para este estudo o idoso em estado de corresidência, aquele que convive com sua família intergeracional (uma ou mais gerações, ou seja, filhos e/ou netos e/ou bisnetos dentre outros) no mesmo domicílio (Camarano et al., 2004).

Além disso, foram incluídos aqueles idosos que possuíam boas condições cognitivas segundo aplicação do Mini Exame do Estado Mental (MEEM). O MEEM fornece informações sobre parâmetros cognitivos avaliando funções como a orientação temporal e espacial, atenção e cálculo, memória, linguagem e capacidade construtiva visual. Com o escore que pode variar de 0 , com maior comprometimento cognitivo, a 30 pontos, com melhor capacidade cognitiva (Lourenço, Veras, 2006). A coleta do estudo aconteceu em duas etapas. No primeiro momento foi identificado o número de idosos cadastrados nas USF através da consulta as fichas de cadastramento familiar (ficha A), sendo feito o levantamento dos idosos que vivem sozinhos e aqueles que corresidem com familiares, com a ajuda dos Agentes Comunitários de Saúde. Em seguida, ocorreu as visitas aos domicílios para aplicação dos instrumentos da pesquisa, onde aplicou-se a entrevista por meio do questionário semiestruturado contendo dados de identificação dos participantes, como idade, sexo e composição do arranjo familiar, depois foi aplicado aos idosos o questionário Brazil old age Schedule (BOAS) adaptado e o questionário de Estado de Saúde. O Questionário Brazil Old Age Schedule - BOAS é um instrumento de pesquisa que investiga fatores multidimensionais do envelhecimento, baseado em outros instrumentos com padrões aceitáveis de validade e confiabilidade. O BOAS foi concebido a partir de 8 grandes categorias (Informações gerais, saúde física, utilização de serviços médicos e dentários, atividades de vida diária, recursos sociais, recursos econômicos, saúde mental, necessidades e problemas que afetam o entrevistado) (Veras; Dutra, 2008). Os dados obtidos através do questionário sociodemográfico, do BOAS e da escala de Estado de Saúde foram tabulados e analisados segundo o The StatisticalPackage for Social Sciences para Windows (SPSS 21.0, 2013, SPSS, Inc, Chicago, IL). Em todas as análises o nível de significância adotado será de $5 \%(\alpha=0,05)$. Este estudo faz parte de uma ampla pesquisa intitulada "Arranjo Familiar de Idosos em Corresidência Residentes em Municípios do Nordeste e Sudeste do Brasil", tendo sido aprovada pelo Comitê de Ética em Pesquisa da Universidade Estadual do Sudoeste da Bahia (CEP-UESB), cujo parecer $n^{\circ} 102.641$, foi exarado observando a Resolução do Conselho Nacional de Saúde (CNS) nº 196/96, vigente na época. Sendo assim, foi encaminhado ao CEPUESB uma solicitação de inclusão deste estudo ao projeto principal de acordo a Resolução $n^{\circ} 466$, de 12 de dezembro de 2012. A produção dos dados foi realizada após a emissão do parecer favorável do CEP-UESB de inclusão do presente campo de estudo, e da autorização da Secretaria Municipal de Saúde de Vitória da Conquista - BA para a realização da pesquisa nas USF. A participação dos idosos foi voluntária, sendo formalizada a aceitação mediante a assinatura do Termo de Consentimento Livre e Esclarecido (TCLE), que assinou em duas vias, sendo uma de domínio do informante e a outra da guarda da pesquisadora responsável pelo estudo.

\section{RESULTADOS}

Dentre a população abordada, após análise de critérios de inclusão e exclusão, totalizou-se 239 idosos estudados. Dentre estes participantes do estudo, a média de idade encontrada foi de $72,2 \pm 8,08$, sendo a idade mínima encontrada 60 anos e a máxima 95 anos. Verificou-se uma maior predominância do sexo feminino (77\%) e do estado civil casado (42,6\%), sendo relevante também a presença de viúvos $(41,8 \%)$. No que diz respeito ao arranjo familiar da corresidência, identificou-se maior convivência com netos $(57,3 \%)$, seguido de filhos (a) (55,2\% do sexo masculino e $54,0 \%$ do sexo feminino), conforme dados da Tabela 1. Quanto ao Estilo de Vida dos idosos, 91,3\% referiram nenhum consumo semanal de bebida alcoólica e, no tocante hábito de fumar, verificou-se que 58,2\% dessa população nunca fumou, destacando-se, porém, que $33,3 \%$ apesar de não ser fumante atualmente, já fumou (Tabela 2). De acordo com os dados condensados na tabela 3, 95,05\% (227) dos idosos corresidentes se dizem satisfeitos com a vida e apenas 3,3\% (8) referiram insatisfação.

\section{DISCUSSÃo}

Este estudo observou que os idosos em corresidência possuem uma idade média de 72,2 anos. Resultado semelhante foi encontrado no estudo de Pinto Júnior et al. (2016), onde os idosos entrevistados possuem idade média de 72,7 anos. 
Tabela 1. Características sociodemograficas e composição do arranjo familiar dos idosos participantes. Vitória da Conquista - BA, 2018

\begin{tabular}{|c|c|c|c|}
\hline Variáveis & $\%$ resposta & $\mathrm{N}$ & $\%$ \\
\hline Sexo & 100 & & \\
\hline Feminino & & 184 & 77,0 \\
\hline Masculino & & 55 & 23,0 \\
\hline Grau de escolaridade & 97,5 & & \\
\hline Analfabeto & & 81 & 34,8 \\
\hline Primário incompleto & & 108 & 46,4 \\
\hline Primário completo & & 28 & 12,0 \\
\hline $1^{\circ}$ grau completo & & 5 & 2,1 \\
\hline $2^{\circ}$ grau completo & & 10 & 4,3 \\
\hline Superior completo & & 1 & 0,4 \\
\hline Estado conjugal & 99,2 & & \\
\hline Nunca casou & & 4 & 1,6 \\
\hline Casado(a)/morando junto & & 101 & 42,6 \\
\hline Viúvo (a) & & 99 & 41,8 \\
\hline Divorciado(a)/separado & & 33 & 13,9 \\
\hline Composição do arranjo familiar & 100 & & \\
\hline Esposo(a)/companheiro(a) & & 97 & 40,6 \\
\hline Pais & & 5 & 2,1 \\
\hline Filhos & & 132 & 55,2 \\
\hline Filhas & & 129 & 54,0 \\
\hline Irmãos/irmãs & & 6 & 2,5 \\
\hline Netos(as) & & 137 & 57,3 \\
\hline Outros parentes & & 16 & 6,7 \\
\hline Outras pessoas & & 13 & 5,4 \\
\hline
\end{tabular}

Fonte: Dados da pesquisa

Tabela 2. Dados referentes ao Estilo de Vida dos idosos. Vitória da Conquista, 2018

\begin{tabular}{lccc}
\hline Variáveis & \% resposta & $\mathrm{N}$ & $\%$ \\
\hline Média de dias por semana que & 86,6 & & \\
consome bebida alcoólica & & & \\
Nenhum & 189 & 91,3 \\
Menos de 1 dia por semana & 4 & 1,9 \\
1 dia por semana & 1 & 0,5 \\
2-3 dias por semana & 10 & 4,8 \\
4-6 dias por semana & 1 & 0,5 \\
Todos os dias & 2 & 1,0 \\
Hábito de fumar & & \\
Fuma atualmente & 18 & 8,5 \\
Já fumou, mas não fuma mais & & 71 & 33,3 \\
Nunca fumou & & 124 & 58,2 \\
\hline
\end{tabular}

Tabela 3. Satisfação de vida dos idosos corresidentes. Vitória da Conquista - BA, 2018

\begin{tabular}{cccc}
\hline Variáveis & \% de resposta & $\mathrm{n}$ & $\%$ \\
\hline Satisfação de vida & 98,3 & & \\
Satisfeito & & 227 & 95,0 \\
Insatisfeito & & 8 & 3,3 \\
Não responderam & 4 & 1,7 \\
\hline
\end{tabular}

Esse dado pode ser reflexo do aumento da expectativa de vida associado a melhores condições sociais e de saneamento básico, estando associado ao controle de doenças e redução da taxa de mortalidade (BARBOSA et al., 2018). Em relação ao gênero houve uma predominância do sexo feminino (77\%). Corroborando com os estudos de Rabelo e Neri (2015) e Medeiros et al. (2018) onde apresentaram em sua maioria mulheres como entrevistadas, com respectivamente $77,6 \%$ e $90,7 \%$ da amostra. Dessa forma, percebe-se que há uma proporção maior de mulheres que homens. Essa feminização da velhice pode estar atribuída a diminuição da exposição das mulheres aos fatores de risco de mortalidade, à maior procura aos serviços de saúde, por apresentarem uma maior precaução com o autocuidado (Silva et al., 2014). Verificou-se em relação ao tipo de composição familiar da corresidência, que os idosos em sua maioria convivem predominantemente com netos (as) (57,3\%), seguido por filhos $(55,2 \%)$ e filhas $(54 \%)$. Estudos de Meira (2014) realizados em Minas Gerais, corroboram com esses resultados, mostrando uma alta prevalência desse tipo de arranjo familiar de idosos com netos e filhos, $(71 \%)$ e $(64,5 \%)$, respectivamente. A corresidência marcada pelo convívio intergeracional, se deve, em grande maioria, ao fato dos idosos serem a única fonte de apoio para os netos e filhos, que obtiveram insucesso na tentativa da vida fora do contexto familiar (Ramalho; Júnior, 2018). Segundo Brito et al. (2019) a dificuldade dos filhos adentrarem no mercado de trabalho ou casos após divórcios e separações, favorecem a instalação do estado de corresidência, onde os idosos que possuem boa capacidade física se tornam cuidadores dos netos, possibilitando que os filhos saíam para trabalhar deixando as crianças em segurança. De acordo Camarano e El Ghaouri (2003), essa relação entre gerações é encontrada com grande facilidade em países em desenvolvimento, como o Brasil, pois, pode ser utilizado como estratégia de sobrevivência para diminuição das despesas. Além disso, a instalação da corresidência pode ocorrer pertinente a necessidade do idoso devido a uma dependência física, tendo em vista que esse tipo de arranjo apresenta benefícios como, a troca de ajuda mútua entre os familiares, lealdade e segurança, fortalecendo assim os laços familiares (Lopes et al., 2015). Entretanto, segundo estudos de Rabelo e Neri (2015) o apoio intergeracional parece ser mais expressivo das gerações mais velhas para as mais novas, uma vez que, os idosos demonstram mais preocupação em relação ao modo de vida dos familiares, podendo assim, ocasionar prejuízos na satisfação e estilo de vida dos idosos corresidentes.

No que se refere ao estilo de vida o estudo mostrou que $(91,3 \%)$ dos entrevistados não consomem álcool em nenhum dia da semana. Outros estudos, como o realizado por Silva et al. (2018), também apresenta um alto índice de idosos não etilistas $(83,6 \%)$. O abuso de álcool nessa faixa etária é caracterizado como uma epidemia invisível, estando associado a indicativos sintomas de problemas psiquiátricos, como a depressão, gerando impactos na saúde, assim, interferindo na satisfação de vida dos idosos (GUIMARÃES; TAVARES, 2019). Segundo estudos de Barreto, Carreira e Marcon (2015), a presença de doenças crônicas, como hipertensão arterial sistêmica e o diabetes mellitus, é um dos fatores que tem relação direta com idosos não etilistas, apontando que, quando o idoso faz uma autopercepção ruim de sua saúde as probabilidades do indivíduo modificar seus hábitos, buscando melhoras para um estilo de vida saudável, são altas. Outro fator que influencia para o baixo consumo de álcool dos idosos é o convívio intergeracional, pois, a família, na visão do idoso, é o lugar onde ele pode depositar a maior expectativa positiva de cuidado. Os familiares oferecem ao idoso um suporte que funciona como uma rede informal, agindo como orientadores e cuidadores da saúde dos idosos corresidentes (Albuquerque et al., 2016). Quanto ao uso do cigarro verificou-se que apesar de não fumarem atualmente, uma parcela notável de idosos, já fumou (33,3\%). Corroborando com os estudos de Viana (2013) que mostrou uma quantidade significativa de ex- fumantes na terceira idade, (32,7\%). De acordo Zaitune et al., (2011), o predomínio de ex fumantes com o aumento da idade, pode estar associado com o surgimento do risco de comorbidades devido ao uso do cigarro, como também, a criação de 
campanhas antitabaco, entre outros. Já em relação a população que nunca fumou, observou-se resultado satisfatório, $(58,2 \%)$. Dados semelhantes são encontrados no estudo de Garbaccio et al. (2017) que mostra alto índice de idosos não fumantes na região centro-oeste de Minas Gerais (82,4\%). Segundo Pedreira et al. (2016), durante a vida, as escolhas do indivíduo poderão contribuir ou afetar o seu envelhecimento, nesse contexto a relação de prevenção de doenças causadas pelo cigarro e a alta preocupação com um envelhecimento e saudável, contribui para esses resultados. Conforme estudos realizados por Ribeiro (2014) a família também é vista como incentivadora para prática de hábitos saudáveis, funcionando como um sistema de apoio para os idosos, possuindo autonomia e conhecimento prático para guiar em ações que visam a prevenção e/ou tratamento de doenças. Esse tipo de suporte pode contribuir de maneira eficaz para a conscientização da saúde física e psicológica do indivíduo, para isso é necessário que seja traçado ideias em comum, pautadas na intersubjetividade e relação de cuidado com o idoso.

Em relação a satisfação de vida, os resultados mostraram que há uma grande satisfação dos idosos (95\%). Estudos realizados por Meira et al. (2015) e Pinto Júnior et al. (2016), mostram que os idosos se sentem satisfeitos com a vida que apresentam, com respectivamente $80,5 \%$ e $85,8 \%$. De acordo Joia e Ruiz (2013), a satisfação com a vida depende de avaliações cognitivas e subjetivas, baseando-se em um critério individual sobre a vida, tratando-se de um estado psicológico que possui profunda ligação entre bem-estar e felicidade. Conforme a literatura, o idoso considera a família como fonte primordial de suporte emocional e cuidado, servindo como base de proteção, mostrando-se felizes com a convivência intergeracional, pois usufruem de benefícios, como à companhia e suporte emocional, influenciando assim, em uma longevidade saudável (SILVA et al., 2014). Entretanto, o estudo de Sampaio et al. (2019) traz que um dos indicativos para uma insatisfação de vida dos idosos corresidentes é a preocupação. Como relatado pelos idosos, um dos fatores marcantes dessa preocupação é a instabilidade financeira dos demais familiares, fazendo com que, a grande maioria dos idosos se tornem provedores do lar, utilizando da sua renda ou benefício para o sustento de todos os membros, porém não sendo o suficiente para prover todas as necessidades. De acordo Santos et al. (2019), o estado de corresidência se torna importante para o idoso, desde que aspectos de âmbito psicológico, social e emocional se relacionem de forma positiva conduzindo-os para uma vida satisfatória.

\section{Conclusão}

O presente estudo permitiu obter através dos resultados, importantes informações sobre os idosos que vivem em estado de corresidência, demonstrando que os entrevistados apresentam um bom estilo de vida, principalmente no que diz respeito a não praticar o consumo do álcool e cigarro, além de possuir uma boa satisfação de vida. Dessa forma, pode-se discutir que o convívio intergeracional de idosos e familiares pode favorecer de forma positiva a vida dos idosos. Porém, há necessidade de uma análise subjetiva com maior compreensão de vários fatores que se relacionam a esse convívio, como questões psicológicas e sociais, que podem interferir neste contexto. Este estudo poderá contribuir com maiores discussões sobre o tema e na efetividade de políticas públicas dentro do contexto do Sistema Único de Saúde (SUS) para os idosos em corresidência, sendo válido ressaltar a necessidade de maiores estudos tendo como foco o convívio intergeracional com maiores amostras e populações, a fim de que possa ser ofertado uma melhor qualidade de vida tanto para os idosos quanto para seus familiares.

\section{REFERÊNCIAS}

Aguiar JE 2007. A experiência da co-residência para idosas em família intergeracional. 2007. Dissertação (Mestrado em Enfermagem) - Setor de Ciências da Saúde, Universidade Federal do Paraná, Curitiba.

Albuquerque TDFP et al. 2016. Arranjos familiares, expectativa de cuidado e envolvimento social de idosos urbanos. Congresso Nacional de Envelhecimento Humano, Paraíba.

Barbosa MA 2018. Family Composition and Funcional Capacity of Coresident Elderly People. International Journal of Development Research, Vol. 08, Issue, 06, pp.21291-21295, Junho.

Barreto MS, Carreira L, Marcon SS 2015. Envelhecimento populacional e doenças crônicas: Reflexões sobre os desafios para o Sistema de Saúde Pública. Revista Kairós Gerontologia, 18(1), pp. 325-339.

Brito JA, Sampaio LS, Lessa RS, Vilela ABA, Santos LA, Silva JOL, Barreto PPM, Sampaio TSO 2019. Financial Income and Life Satisfaction of the Elderly Intergenerational Relations. International Journal of Advanced Engineering Research and Science (IJAERS) [Vol-6, Issue-12, Dec- 2019].

Camarano AA, El Ghaouri SK 2003. Família com idosos: ninhos vazios? Rio de Janeiro: IPEA, (Texto para discussão $\mathrm{N}^{\circ}$ 950).

Camarano AA, Kanso S, Mello JL, Pasinato MT. 2004. Famílias: espaço de compartilhamento de recursos e vulnerabilidades. In: CAMARANO, A. A. Os Novo Idosos Brasileiros: Muito Além dos 60? Rio de Janeiro: IPEA, 2004, p. 137-65, 2004.

Garbaccio JL, Tonaco LAB, Estevão WG, Barcelos BJ. 2018. Aging and quality of life of elderly people in rural areas. Rev Bras Enferm [Internet]; 71(suppl 2):724-32 [Thematic Issue: Health of the Elderly].

Guimarães MSF, Tavares DMS. 2019. Prevalence and factors associated whit abuse and likely dependence of alcohol among elderly. Texto contexto enferm. vol.28 Florianópolis.

Horta ALM, Ferreira DCO, Zhao LM. 2010. Envelhecimento, estratégias de enfrentamento do idoso e repercussões na família. Rev. bras. enferm., Ago. Vol.63, no.4, p.523-528. ISSN 0034-7167.

Joia LC, Ruiz T 2013. Satisfação com a Vida na Percepção dos Idosos. Revista Kairós Gerontologia,16(6), pp.79-102. São Paulo (SP), dezembro.

Lopes CRS, Cardoso JP, Meira SS, Casotti CA, Vilela ABA, Pereira HBB (2015). Meaning of co-residence in elderly vision: A strategy for cognitive analysis with use of semantics networks. Rev.Saúde.com 2015; 11(2): 174-182.

Lourenço RA, Veras RP. 2006. Mini-Exame do Estado Mental: características psicométricas em idosos ambulatoriais. Rev. Saúde Pública, v.40(4), 712-719.

Medeiros AOM. 2018. Analysis of the functional capacity of elderly at a clinical school of physiotherapy of a higher education institution: retrospective study. Fisioterapia Brasil ISSN Impresso 1518-9740, v. 19, n. 5. 
Meira SS, Vilela ABA, Casotti CA, Anjos ACF, Silva DM 2014. Considerations about life conditions of elderly in Jequitinhonha Valley, Minas Gerais - Brazil. Serviço Social em Revista, Londrina, V. 17, N.1, P. 159-177, jul/ dez.

Meira SS, Vilela ABA, Casotti CA, Nascimento JC, Andrade CB. 2015. Elderly in the state of co-residence in a town in State Bahia, Brazil. O Mundo da Saúde, v. 39, p. 201-209. São Paulo - 2015.

Pedreira RBS, Andrade CB, Barreto VGA, Pinto Junior EP, Rocha SV. 2016. Autopercepção de saúde entre idosos residentes em áreas rurais. Revista Kairós Gerontologia, 19(1), pp. 103-119. São Paulo (SP), (janeiro-março).

Pinto Junior EP, Silva IT, Vilela ABA, Casotti CA, Pinto FJM, Silva MGC. 2016. Functional dependency and associated factor in elderly living with relatives. Cadernos Saúde Coletiva, Rio de Janeiro, 2016 v. 24, p. 404-412.

Rabelo DF, Neri AL. 2015. Family configuration and physical and psychological health status in a sample of elderly. Cadernos de Saude Pública, Rio de Janeiro, v. 31, p. 874884, abr, 2015.

Ramalho HMB, Júnior JLSN 2018. Intergenerational mobility of education and co-residence decision between adult children and parentes in Brazil. Análise Econômica, Porto Alegre, v. 36, n. 69, p. 231-266, mar.

Ribeiro JF (2014). Autonomia do ser idoso: percepção da família corresidente. Dissertação de mestrado. Universidade Estadual do Sudoeste da Bahia Departamento de Saúde Programa de Pós-graduação em Enfermagem e Saúde, Jequié-BA, 2014.

Sampaio TSO, Vilela ABA, Sampaio LS (2019). Representational content and structure about family from the viewpoint of co-residency elderly people. Ciência \& Saúde Coletiva, v. 24, p. 1309-1316, 2019.

Santos LA, Sampaio TSO, Lessa RS, Brito JA, Vilela ABA, Barreto PPM, Reis LA, Sampaio LS. 2019. Association between Income and SelfPerception of Health with Life
Satisfaction of life of Seniors in Corresidence. International Journal of Advanced Engineering Research and Science (IJAERS) [Vol-6, Issue-12, Dec - 2019].

Silva AA. 2016. Fatores associados a funcionalidade familiar de idosos na estratégia Saúde da família. [Dissertação]. Fortaleza (CE): Universidade Federal do Ceará, 2016 /Alyne Andrade Silva.

Silva DM, Vilela ABA, Nery AA, Duarte ACS, Alves MR, Meira SS. 2015. Dinâmica das relações familiares intergeracionais na ótica de idosos residentes no Município de Jequié (Bahia), Brasil. Ciênc. Saúde Coletiva vol.20 no.7 Rio de Janeiro July.

Silva IT, Pinto Junior EP, Vilela ABA. 2014. Self concept of health of elderly living with relatives. Revista Brasileira de Geriatria e Gerontologia, Rio de Janeiro, 2014, v. 17, p. 275-287.

Silva PAB, Santos FC, Soares SM, Silva LB. 2018. Perfil sociodemográfico e clínico de idosos acompanhados por equipes de Saúde da Família sob a perspectiva do gênero. Rev Fund Care Online. Jan./mar.; v.10 p.7-105.

Torres IS, Sampaio LS, Silva FF, Reis LA, Barbosa MS, Soares IS, Lessa RS, Vilela ABA, Bittencourt ASL, Samapio TSO (2018). Perception od elderly people in cohabitation regarding their family relatioships. International Journal of development Research, 8, (12), 24526-24531.

Veras R, Dutra S. 2008. Perfil do idoso brasileiro: questionário boas. Rio de Janeiro: UERJ/UnATI.

Viana DA 2013. Influência do tabagismo na qualidade de vida de idosos da comunidade. Dissertação de Mestrado em Atenção à Saúde - Universidade Federal do Triângulo Mineiro, Uberaba.

Zaitune MPA 2010. Comportamentos de saúde de idosos: Resultados de inquérito populacional. Disponível em: https://scholar.google.com.br/citations?user=DgnHgyAAA AAJ\&hl=pt-BR. [Acesso em 22 de maio de 2020]. 